\title{
Interdependence Days
}

July is the month in which two historically linked nations, France and the United States, celebrate freedom. I was recently afforded the privilege of acting as one of the representatives of the Materials Research Society (based in the United States) to the European-MRS meeting held in Strasbourg, France. It was a pleasure to share the spirit of international cooperation and free exchange of ideas that is a hallmark of E-MRS activities. I was also struck by the degree to which materials research, especially as presented at the meeting, is associated with freedom as an ideal.

The E-MRS program was highlighted by three extraordinary plenary lectures. All three speakers described how they and their colleagues aim to overcome fundamental limitations and to break barriers to the miniaturization of electronic devices, chemical processors, and optical waves. First, Siegmar Roth, of the Max Planck Institute in Stuttgart, spoke about potential applications of carbon nanotubes. Next, Thomas Ebbesen, of the nearby Université Louis Pasteur in Strasbourg, discussed the fascinating optical properties of arrays of narrow apertures in thin metal films. The concluding talk was given by Wolfgang Mehr, of IHP in Frankfurt, about alternatives to conventional silicon-based electronic materials and processes. In all three cases, it was clear that the researchers were striving to surpass - to free us from, if you willconstraints imposed by assumed physical laws. For example, the greatly enhanced and selective transmission of electromagnetic energy through seemingly impenetrable media described by Ebbesen, and the emission of electrons (with subsequent production of photons) from individual nanotubes, deemed one of the more promising capabilities of such structures, are approaches to overcoming the bounds of diffraction seemingly imposed by the laws of optics. Higher-performance displays and signal processing systems are envisioned from these nanomaterials

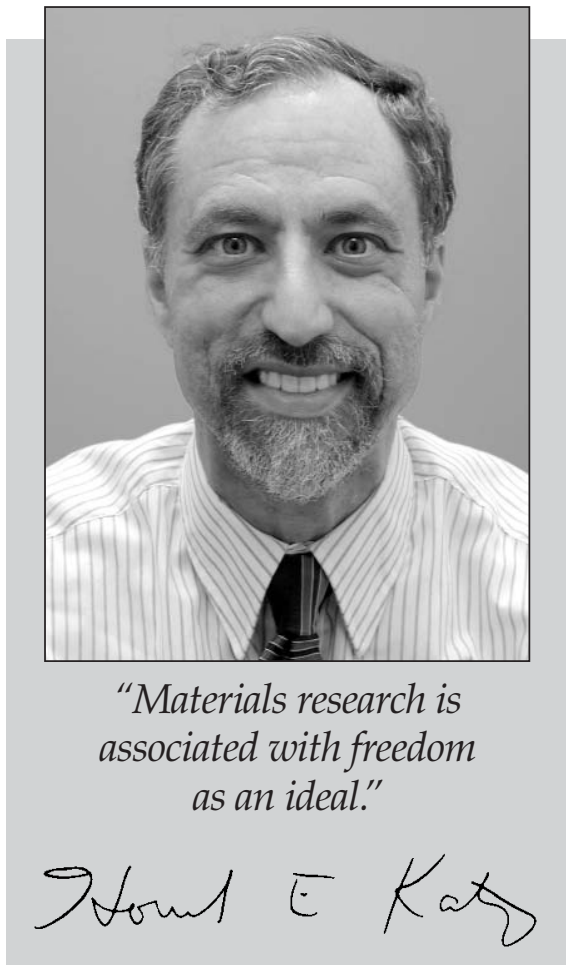

advances. The alternatives considered by Mehr, including silicon/germanium hybrids, copper leads, heavier metal oxides, and photonic circuits with silicon emitters, are potentially a suite of innovations that would collectively allow the increases in circuit speed projected by Moore's law to continue beyond what is believed to be physically possible on integrated circuits as currently conceived.

In addition to our desire to escape from fundamental and microscale physical limits, we as global citizens are compelled to free ourselves from macroscopic limits, and even threats, to human development. On one hand, the plenary speakers cautioned us to resist the temptation to exaggerate the near-term impact of exotic materials on pressing societal problems, especially when the materials have not yet been fully characterized and evaluated. For an example, the hydrogen storage capability of carbon nanotubes, according to Roth, probably cannot exceed that of a conventional pressure vessel. The roadmap for the insertion of single-electron and single-molecule concepts into silicon-type platforms, according to Mehr, reaches far into the future. There are also new sustainability and ecological issues arising from the proposed wider use of new materials, especially nanostructured ones.

On the other hand, much of the E-MRS technical program was in fact focused on pressing societal technology needs, especially the need to become independent of a diminishing supply of fossil fuels. Many presentations were devoted to fuel-cell and solar-cell materials, ranging from solid-oxide conductors to compound semiconductors. One notable invited talk was delivered by Shigeru Niki from the National Institute of Advanced Industrial Science and Technology (AIST) in Ibaraki, Japan. He gave a detailed and well-reasoned account of several new analytical techniques that are being employed for in situ optimization of highly complex semiconductor thinfilm compositions and deposition conditions. He opened his lecture with a spectacular illustration: the technology park where he is based. Many of the buildings, including the parking structure and the bus station, are covered with solar panels, making this the largest solar town in Japan. In fact, $20 \%$ of the area of the site is solar-panel-coated! This admirable dedication to a pressing societal need-and a demonstration of what the solution could look like-represents the best that materials science can offer, and is the face of materials research that our stakeholders expect and deserve to see. As our fireworks celebrate the freedoms we enjoy now, let us continually enlist materials research in pursuit of the freedoms of the future.

HowARD E. KATZ 2004 MRS President

\section{Over 11,000 Proceedings Papers Now FREE Online For MRS Members}

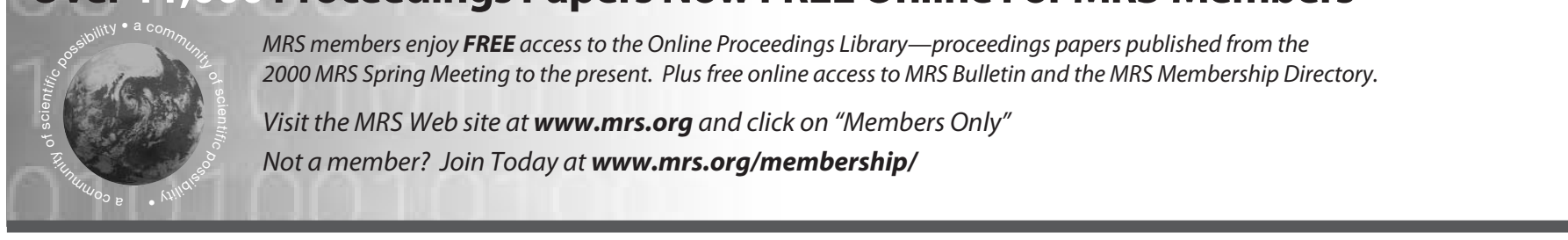

